\title{
Cultural, Emotional, and Associative Traits to Determine Literary Inclination
}

\author{
Hrishikesh Kulkarni ${ }^{1}$, Bradly Alicea ${ }^{2,3}$
}

\begin{abstract}
Various cultural and emotional preferences delineate human creativity. One such set of preferences, literary inclination, impacts not only the books people choose to read but also on the way they perceive the narrative and human relationships contained within. Understanding the overall development of a plot and the evolution of relationships among different characters has significant implications for holding the reader's attention. In this paper, we establish a computational means to derive progressions and associations among characters in a given narrative. We use two books from different cultural traditions (American popular literature and Indian Marathi literature) to validate this technique. For purposes of measuring relationships progression between different characters, we propose the Graphical Association Method (GAM). Further analysis of changes in these imaginary social relationships in relation to a reader's literary inclinations demonstrates that this method holds promise for a more general analysis of narrative structure.
\end{abstract}

Keywords: Machine Learning, Information Retrieval, Natural Language Processing, Graphical Association Method, Narrative Processing, Cross-Cultural

\section{Introduction and Related Work}

As a member of a book clubs, I have observed different opinions of readers and listened creative conversations among them. The journey from mere communication to conversation is core to the human experience. When readers converse, it leads to the generation of many new concepts. This paper is motivated by a simple observation from responses of readers to different creative artifacts. While over two million books are published every year, any given reader will become invested in only about a dozen or so titles. Selecting a book to read depends on a number of factors, including topic, author, and most importantly on the interpersonal relationships between characters as the narrative progresses.

This concept evolved with my three years of interaction with book lovers. There are a few researchers who gave a serious consideration to similar concepts. It has two aspects: one is profiling readers, and another is understanding progression of relationship among characters in different narratives. Culture and emotions are core parts of reader profiling. Cultural attributes are determined based on expressions. There is strong relationship between culture and expressions (Mooij et al., 2011). The way culture and behavioral patterns are associated, it helps in systemic profiling (Henrich, 2015). In the same way culture has association with reading inclination. When we talk about narratives, we are actually referring to the relationship between characters.

Changes in cultural attributes over time can be crucial to understand the narrative (Mohit et al., 2016). Relationship states and trajectories are key in determining these changes. These

\footnotetext{
${ }^{1}$ Computer Engineering Department, PVG’s COET, SPPU, Pune, INDIA hrishikeshparag@gmail.com

${ }^{2}$ Orthogonal Research and Education, Champaign, IL USA bradly.alicea@outlook.com

${ }^{3}$ Open Worm Foundation, Boston, MA USA
} 
relationships can be modeled using semi-supervised machine learning (Snigdha et al., 2016). Changes in these relationships can be represented as patterns. Dynamic relationship identification can be used on social media data. In this whole process there is need to understand narrative structure (Snigdha et al., 2015). Here, it is important to understand change in sentiment (Li et al., 2018). This progress in relationships is also associated with overall progress in story within the boundaries of the plot. This has been dealt with using a variety of quantitative constructs. For example, topical consistency and coherence are crucial in determination of topic transitions (Lund, 2018). In addition, narratives and associated fictions are based on series of events. The relative occurrence of events and their association has an impact on sentiments (Dandan et al., 2017).

We must also take into account linguistic perspectives. There are surprises in overall story progressions. These surprises are also applicable to progressions in relationships. Surprises bring changes in relationships and in fact differentiating adorable events in story (Oard et al., 2019). Thus, for a story multiple events and abstractive surprise elements could prove to be key, where abstractive summarization can be very useful (Chen \& Bansal, 2018). This overall narrative can be viewed as emotional journey. There are emotional aspects associated with part of story depicted through different sentences (Quan et al., 2011; Fotis et al., 2012). When multiple plots are under consideration sentence similarities can be used at preliminary state (Saad et al., 2013). At the next level it is more about sentiment composition and progression (Mitchell et al., 2008). This sentiment analysis can be done from multiple perspectives (Liu et al., 2010).

We take the perspective that creative artifacts (in this case single books) need to be analyzed from a variety of perspectives (Berendt et al., 2019). One such perspective is discourse analysis. Discourse analysis does not simply involve analyzing conversations, but rather the emotional exchange conversations provide among actors (Karthik et al., 2015). Various computational discourse analysis methods have also been proposed (Althoff et al., 2016). One of these methods involves dispute detection and discourse analysis (Wang et.al., 2014), particularly as it relates to personal insult detection. Similarly, the impact of text-based online conversation can be analyzed based on various factors (Rosenthal et al., 2012). In all these scenarios, it is important to choose an analysis which is compatible with cultural analysis and allow for the formation of personality vectors (Kulkarni et al., 2019a; Kulkarni et al., 2019b).

Emotions, expressions, and culturally-relevant touchstones form the basis of a narrative. For example, every character comes with their own set of values and personality. In a typical narrative, multiple characters meet and interact over time. Narrative structure also establishes relationships among different characters. There are strong and weak relationships. As the story progresses, there is likewise a progression of relationships among characters. The progression of these relationships and surprises in these progressions relative to the inclination of a reader form the basis of this study.

Introduction and the Related Work section provide an overview of work carried out in culture modelling, literary text and sentiment analysis and emotional impact of surprises. The Proposed Model section presents the details of a ML model for deriving relationships among characters, clustering narratives with similar progression of relationships and mapping them to personality and emotion vectors of readers. The Data Preparation section includes a proposed mathematical model and data used and prepared for experimentation. The Results and Conclusion sections demonstrate and elaborate upon the results obtained using this model and tries to throw light on possible future directions and applications of this research. 


\section{Proposed Model}

There are multiple factors those decide my literary inclination. Those include cultural makeup, personal emotional traits and social associations.

Hypothesis A: Literary inclination of an individual is based on her/his cultural makeup and emotional traits.

Hypothesis B: The relationship progression among characters in a narrative impact substantially on the preferences of readers.

Our methodology is divided into five important phases:

1. Emotional trait analysis and cultural analysis to determine personality vector of the reader.

2. Narrative Analysis, which involves identification of characters and their actions.

3. Deriving relationships among different characters.

4. Deriving these relationships at regular intervals.

5. Identifying the progression of relationships.

Characters of importance are identified based on the frequent occurrence having maximum association with other characters. Disambiguation and co-reference resolution are done prior to identification of core characters. Typically for example we have chosen two interesting fictions those were read by 50 out of 150 book lovers from the club. It included the book Rage of Angels by Sidney Sheldon and Kraunch Vadh by Marathi writer V.S. Khandekar. We selected these two novels is popularity of these novels among the book lovers we encountered. We have defined core characters in very simple way - a core character is one that has maximum occurrence and fair distribution of presence across the narrative.

Thus, core characters are identified in first phase. This is followed by pivot point selection where major occurrence and interactions among these characters of interest take place. Pivot points are defined as a point of intense interaction associated with the presence of core characters. Emotional upheavals involving the core characters are particularly interesting in terms of pivot point distribution across the narrative.

For example, in Rage of Angels Jenifer Parker, Michael Moretti and Adam Warner are identified as core characters based on occurrence and distribution across the story. While there are a few characters like Abraham Wilson, those have frequent occurrence in some parts of novel, but they are not core since are not distributed and have not expected frequent occurrence. Similarly, Characters like Sulu, Dilip, Bhagvantrao and Dada Saheb are key characters from Kraunch Vadh.

Our methodology is based on extraction of relationships among characters with reference to context of story and cultural significance. This includes pivot point identification, extracting relationship progression at a particular event. These relationships are progressed from one pivot point to next one. This progression is represented on a timeline using graphical 
representation. It can also be represented in matrix form, but we have selected graphical representation because of some of the prominent properties of the same. We even allowed more than two nodes on a single edge. Here graph edge represents local context and nodes on that edge are the core characters present in that context.

To further elaborate approach - with coreference resolution [24] we find the occurrence weight of 27 at pivot point on page 57-58 where Dilip meets Sulu

It's peak is at the exclamation of Dilip "I had attraction outside to come out of Jail - one of my mother and other of..." he points out to beautiful image of Sulu in the mirror. -Page 87 Bhagvantrao and Sulu

Preparing data of book lovers is another key part of this work. We focused on capturing relevant data by presenting questionnaires to these book lovers. For data preparation, key questions are identified those can depict cultural traits. These questions are prepared with multiple revision and under supervision of psychologist and computational social science and literary researchers. The answers to these questions are collected from social network interactions and other communications from each candidate.

While preparing the data, key questionnaires are formed with psychologist and cultural experts. Twenty-two (22) questions are prepared with the input of experts, common issues in the literature, and our specific objectives. The work of cultural clustering is enhanced with literary contextual insights. Here we involved literary experts while preparing questionnaire. Responses to questions are captured in the form of images in selected cases and MCQ options or descriptive text in rest of the cases. While preparing data, the following issues were taken into consideration:

- The data is represented in scattered plot and Manual verification carried out before entering data into dataset.

- In first phase to check validity of questions having indirect reference to literary association - the expression-based approach, answers from eight book lovers are selected and different experiments are carried out on this initial set. These experiments included clustering book lovers and mapping them a book from a sample set those were previously presented at the club.

- In the next phase, responses from 100 book lovers are captured and next level validation is performed.

To analyze the survey data (MCQs), $k$-means method is used to check manual closeness with Euclidean distance measure. Data pre-processing done for MCQ and Textual inputs. The number of clusters are decided based on the data distributions (elbow and silhouette methods are used for this purpose). This method ensures that the data collected can be clustered based on similarities.

For textual data filtering, Nonlinear Programming (NLP)- based pre-processing was done. A manual association based on MCQ relevance is conducted in the given context. These contextual features are then used to generate graphical models. A Principle Component Analysis (PCA) is then used to get association and their validation between selected features. Finally, an association graph matrix is generated from MCQ data. In this phase every book lover is 
represented as a graph Figure 1: Typical parallel weighted relationship where cultural, literary and emotional attributes are represented as nodes. These graphs are clustered using Graphical Association Method. Thus, the proposed algorithm focuses on text data processing to derive cultural association with reference to literary inclination. It consists of four major parts. The algorithm is given below:

- derive context (Personal Context): To derive the intent of candidate the answer-text is pre-processed, and natural language processing is used to determine the topic. The questions with reference to scenarios are considered here. These topics are associated to derive the context.

- $\quad$ association to derive intent: In the next stage similarity among text artefacts and overall intent is determined using hybrid techniques. Two basic methods used for initial association are term distance and the use of LSTM dictionary-based word association.

- Similarity Score: The similarity score is determined using closeness factor. The similarity score and association are used to generate Graphs. In these Graphs weights are generated for text inputs.

- LSTM based word association is used to create weighted graph.

- WordNet association is considered for the same.

○ Cultural association: Each candidate's cultural parameters are represented as a graph.

Every graph has a core theme. The association among graphs is determined using node based graphical association method where the corresponding node among two graphs are determined. Further, with reference to core theme the association among two graphs is determined. Thus, the overall Analysis is performed using graphical and data analysis. Here, Association among data can be divided into three parts:

i. MCQ data Graph,

- Cultural similarities are determined using mapping of MCQ data graphs. Thus, a chronological cultural context is set.

ii. Textual data graph,

○ Cultural similarities using textual graph are mapped. These similarities are quantified in numbers.

iii. Textual similarities

- Cultural similarities among candidates are determined using text vector similarities

\section{Relationship Classification}

The relationship at a pivot point is classified into two basic types viz romantic and general. Romantic relationships are classified into three types: romantic positive - i.e. two characters are in romantic relationship and it is improving, romantic negative, romantic stable. The degree of positivity is decided using NLP and sentiment analysis. We have identified 10 pivot points across the novel for characters Sulu and Dilip namely on pages. Similarly, there are 8 pivot points for Sulu and Bhagwantrao... The progression of their relationships and their association is depicted in Figure 1. 


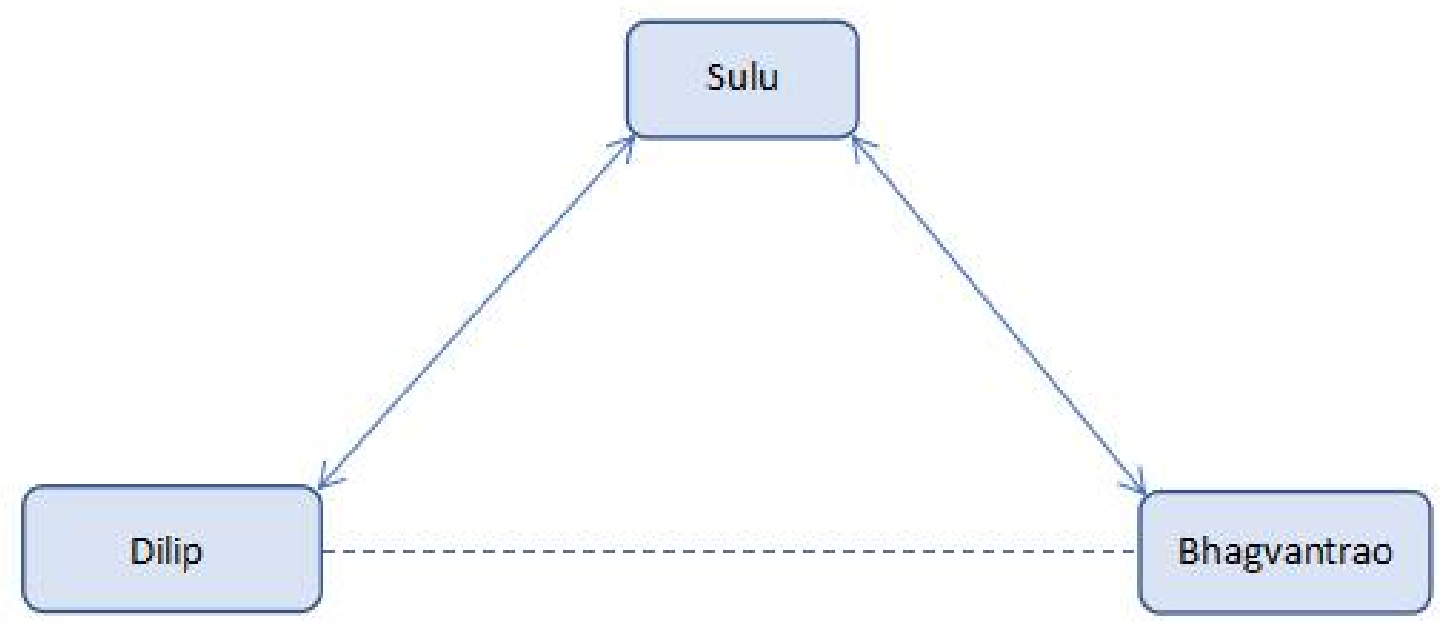

Figure 1. Typical parallel weighted relationship

Aside from the definition of pivot points, other parameters are also considered, and are based on a Euclidian distance.

- $\quad$ core character selection.

- $\quad$ pivot point identification.

- relationship classification and quantification.

- pattern classification.

In the core character selection phase, the most frequently occurring characters are selected. The relationship between these characters are based on the following attributes:

- ranking the sentences occurred in association with two characters in the proximity of pivot point.

- determine the direction of sentence like Jenifer to Adam or Adam to Jenifer.

- $\quad$ sequence them.

- $\quad$ give weight to every sentence and classify the feelings.

- the slope of the graph tells us emotional inclination (it also includes relation polarity).

Pivot point identification:

Definition: Narrative pivot point is one where more than one core characters are present and dialogues are depictive of relationships. Figure 4 depicts the progression of relationship between Jenifer and Adam and Jenifer and Michael in Rage of Angels. 


\section{Data Preparation}

In any cultural studies and intelligent methods for associating cultures data is the key. All machine learning techniques are data hungry and it becomes even more critical when we are dealing with cultural and emotional aspects of humans and mapping it to stories in the books. To derive personality vector for book lovers we have prepared a questionnaire. The profile and answers to these questions are used to prepare personality vector for every reader. These personality vectors are clustered and mapped to patterns of character relationship progression.

- it should be interesting: The candidate should love to answer these questions.

- $\quad$ easy to answer: Candidate will not have to take pain to answer it.

- relevant but not direct: The information should be derived from indirect clues since direct answers are mostly misleading.

- make the candidate think: It should elicit a strong emotional response.

Twenty-two (22) questions are considered which do not contain any personal information. Data is prepared that can help in decoding context based on behavior of candidates. Attribute considered are depicted in table 1. Filtering of data is done which is later converted into numeric and non-numeric data. Filtering is done on text data as stop word removal, stemming and other methods are applied for cleaning and filtering the data. Table 1 shows parameters used to build context.

The previously defined k-means clustering method is used to validate the thought process of feature creation. Initial data are captured, and a $k$-means analysis is conducted. Our analysis then proceeds as follows:

1) selection of number of clusters is done to express the categories.

2) optimal number of clusters defines number of groups that can be created.

3) optimal number of clusters are selected using elbow and silhouette method.

Table 1. Attributes to build context.

\begin{tabular}{|l|l|l|l|}
\hline \multicolumn{1}{|c|}{ Recent Read } & Narrative outcome & Book association & \multicolumn{1}{c|}{ Traveling attributes } \\
\hline $\begin{array}{l}\text { Behavior with } \\
\text { reference situation }\end{array}$ & Work Information & Social attributes & $\begin{array}{l}\text { Week days and weekend } \\
\text { routine }\end{array}$ \\
\hline Hobbies & Events & Friends & \\
\hline
\end{tabular}

Clusters are formed using numeric data of MCQs based on options selected. Let us assume that two people having nearly same traveling and personal attributes are represented by the same cluster. In this case, the distance between them should be minimal (e.g. asymptotic to zero). The following measure represents the sum of intra-cluster distances between points in a given cluster $C_{k}$ containing $n_{k}$ points. Adding the normalized intra-cluster sums of squares gives a measure of cluster compactness

$$
W_{k}=\sum_{k=1}^{K} \frac{1}{2 n_{k}} D_{k}
$$


Personalized dictionary is created for designing graph based on the data captured. A context graph is then created, and weights are assigned, in addition to generation of a graphical model demonstrating a star topology. Each text key vector is connected to other context vectors.

Semi-Graph: Semi-graph is represented as $V$ vertices $X$ set of ordered $n$-tuples, called edges of semi-graph of distinct vertices, for various $n>=2$, satisfying the following conditions:

SG1: Any two edges have at most one vertex in common.

SG2: Two edges $E_{1}=\left(\mathrm{u}_{1}, \mathrm{u}_{2}, \ldots, \mathrm{u}_{\mathrm{m}}\right)$ and $E_{2}=\left(\mathrm{v}_{1}, \mathrm{v}_{2}, \ldots ., \mathrm{v}_{\mathrm{n}}\right)$ are considered to be equal if and only if three conditions hold: 1) $m=n, 2) u_{i}=v_{i}$ for $\left.1 \leq i \leq n, 3\right) u_{i}=v_{i+1}$ for $1 \leq i \leq n$.

Representative Core Character (RCC) involves the core character in narrative, and is derived from associations of two or more characters. When more characters get associated with the character of interest, the weight associated with it increases.

Equation 2 gives mathematical definition of RCC.

$$
\begin{gathered}
\forall c \in c \mid C \in[C C] \\
c \rightarrow[C C]
\end{gathered}
$$

where $[\mathrm{CC}] \neq 0$. Going through narrative in an iterative fashion, the core characters are identified. The characters Jenifer, Michael and Adam are core characters in Rage of Angels, while Kraunch Vadh features core characters Sulu, Dilip, and Bhagvantrao.

The association among personality vectors helps in clustering book lovers. These clusters have different properties. In very interesting analysis we have begun with larger number of clusters and slowly based on literary inclination the clusters are combined. It's slightly different experiment than typical supervised learning. The closeness among two characters is derived based on occurrence of words in the communication between two characters. We determined it using covariance between two vectors.

Let $n$ be number of features associated with book lover. For all book-lovers personality vector is defined as

$$
V P=\left[\begin{array}{c}
V P_{1} \\
V P_{2} \\
V P_{3} \\
\vdots \\
V P_{n}
\end{array}\right]
$$

Similarly, a book vector can be derived mathematically and based on the relationships among characters 


$$
V T=\left[\begin{array}{c}
V T_{1} \\
V T_{2} \\
V T_{3} \\
\vdots \\
V T_{n}
\end{array}\right]
$$

The distance between two vectors is defined using covariance between them. Let value of Personality Vector (VP) at time $t$ is $\mu_{t}$ and value of Book Vector (VB) at time $t$ is $\gamma_{t}$. Joint variability of vectors helps to define association between them. We used covariance for the same. Thus, covariance between these two vectors at time $t$ is defined as shown in Equation 5.

$$
\operatorname{Covar}[V B, V P]=E\left[\left(V B_{A}-\mu_{t}\right)\left(V P_{A}-\gamma_{t}\right)\right]
$$

Here $V B_{A}$ and $V P_{A}$ are values of VS and VP respectively at initial state. The book progresses hence this progression is represented as a state diagram. Let expected value of Scenario Vector (VS) at time $t$ is $\alpha_{t}$ and expected value of Team Vector (VT) at time $t$ is $\beta_{t}$. Then covariance between these two vectors at time $t$ is defined as shown in Equation 6 .

$$
\operatorname{Covar}[V S, V T]=E\left[\left(V S_{A}-\alpha_{t}\right)\left(V T_{A}-\beta_{t}\right)\right]
$$

Here $V S_{A}$ and $V T_{A}$ are actual values of VS and VT respectively. Hence correlation between VS and VP using standard deviation is defined as follows in Equation 7.

$$
\rho(V S, V P)=\frac{\operatorname{Covar}(V S, V P)}{S D(V S) S D(V P)}
$$

Similarly correlation between VS and VT using standard deviation is defined as follows in Equation 8.

$$
\rho(V S, V T)=\frac{\operatorname{Covar}(V S, V T)}{S D(V S) S D(V T)}
$$

Finally, a variant of the Pearson correlation can be used to calculate contextual closeness between these two vectors. This is defined in Equation 9 in the following manner.

$$
\hat{p}\left(V S_{i}, V S_{j}\right)=\frac{\operatorname{Covar}_{\text {Pearson }\left(V S_{i}, V S_{j}\right)}}{\sqrt{\operatorname{Covar}_{\text {Pearson }}\left(V S_{i}, V S_{i}\right) \operatorname{Covar}_{\text {Pearson }}\left(V S_{j}, V S_{j}\right)}}
$$




\section{Results}

Book lovers are clustered based on cultural and emotional traits. This cultural and emotional association among book lovers has relationships with book they choose and even the part of book they liked the most. The clustering of 150 book lovers is done in four clusters. Interesting observation is majority of book lovers in a particular cluster have affinity to a particular pivot point over other pivot points. In Rage of Angels out of 10 pivot points cluster 1 readers have major affinity to pivot point 4 . Average ranking for different clusters for various pivot points is depicted in Table 2 .

Table 2. Pivot point mapping and ranking.

\begin{tabular}{|l|l|l|l|l|}
\hline Pivot Points & Cluster 1 & Cluster 2 & Cluster 3 & Cluster 4 \\
\hline 1 & 4 & 5 & 1 & No \\
\hline 2 & No & 4 & No & No \\
\hline 3 & No & No & No & No \\
\hline 4 & 1 & 3 & 2 & No \\
\hline 5 & 2 & 2 & 3 & No \\
\hline 6 & 3 & 1 & 4 & No \\
\hline 7 & No & No & 7 & No \\
\hline 8 & 5 & No & No & No \\
\hline 9 & 6 & 6 & 5 & No \\
\hline 10 & No & 7 & 6 & No \\
\hline
\end{tabular}

Figure 2 and Figure 3 depicts the character relationship progression in Kraunch Vadh. The cultural and personality vectors of readers are mapped to these representative progressions. We find the relationships getting stable over the time in Kraunch Vadh. The relationships between Sulu and Dilip has some sudden changes at pivot points 4 and 5 and as per readers probably that is the most attractive part and impacted on overall inclination to read this book. When we applied the same algorithms of pivot point identification and relationship progression determination, we found relationships in Rage of Angels are more distributed.

Specifically, the impact of evolution for another relationship on the original one is minimal. To examine this have also identified pivot points for the relationship between Jenifer and Adam and additional pivot points for the relationship between Jenifer and Michael. The relationship between pivot points for the Jenifer-Adam and Jenifer-Michael relationships is shown in Figure 4. As there is no overlap between these sets of relationship pivot points, there is a need for additional pivot point association outside of the dyadic relationships presented here.

Overall, the results suggest that this method can be applied successfully to other titles. Returning to Figure 4, we can demonstrate how this analysis might be improved to show additional relationships. For example, the pivot point 4 is representative of relationship that is liked by book lovers from cluster 1 . Similarly pivot point 6 is representative relationship of interest for cluster 2. Meanwhile, the narrative progression through pivot points 4-5-6 is of interest for majority of book lovers. It is interesting to see the results when the work is extended to larger book corpus and a greater number of book lovers. 


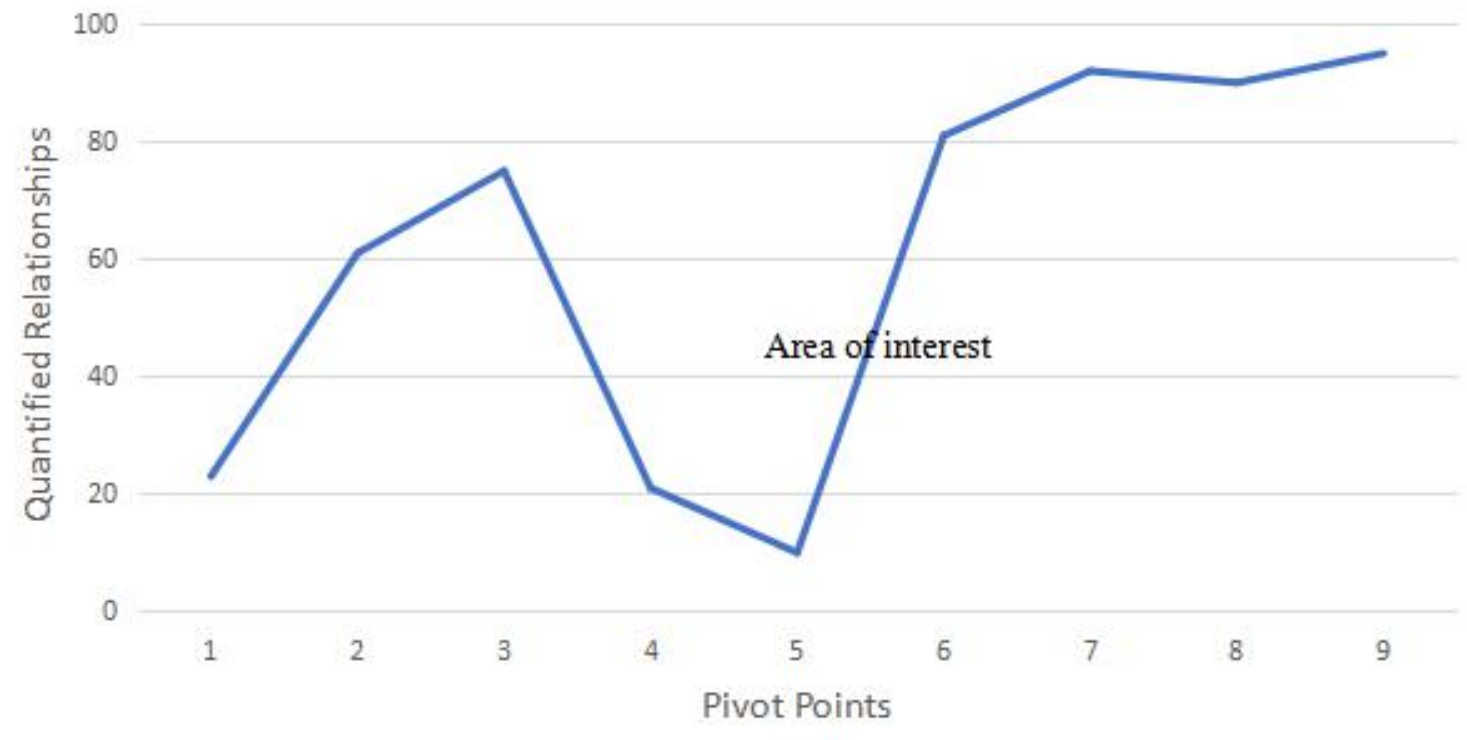

Figure 2: Character relationship progression of Dilip and Sulu in Kraunch Vadh

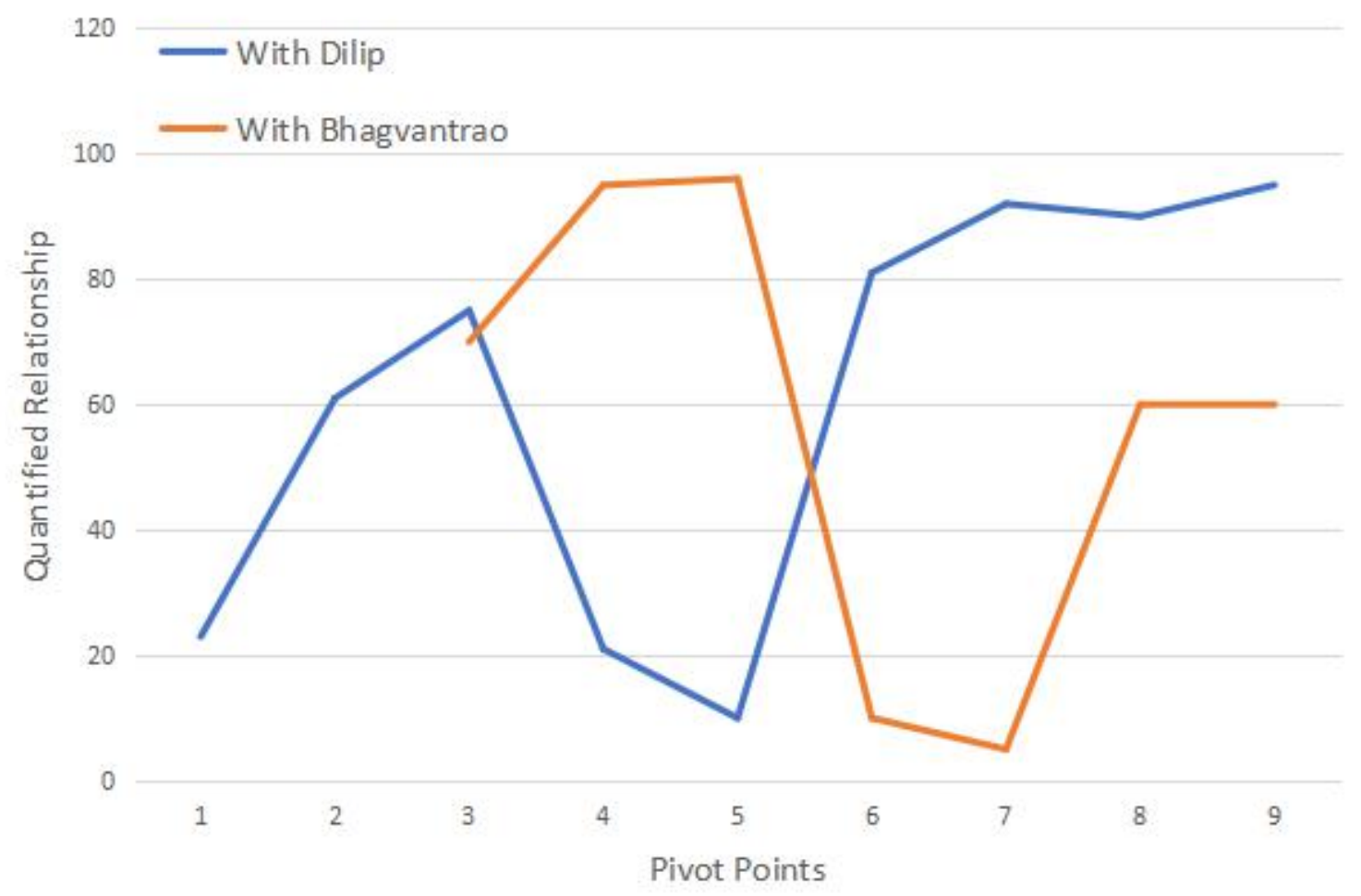

Figure 3: Multi-character relationship progression of Sulu with Dilip (blue) and Sulu with Bhagvantrao (orange) in Kraunch Vadh. 


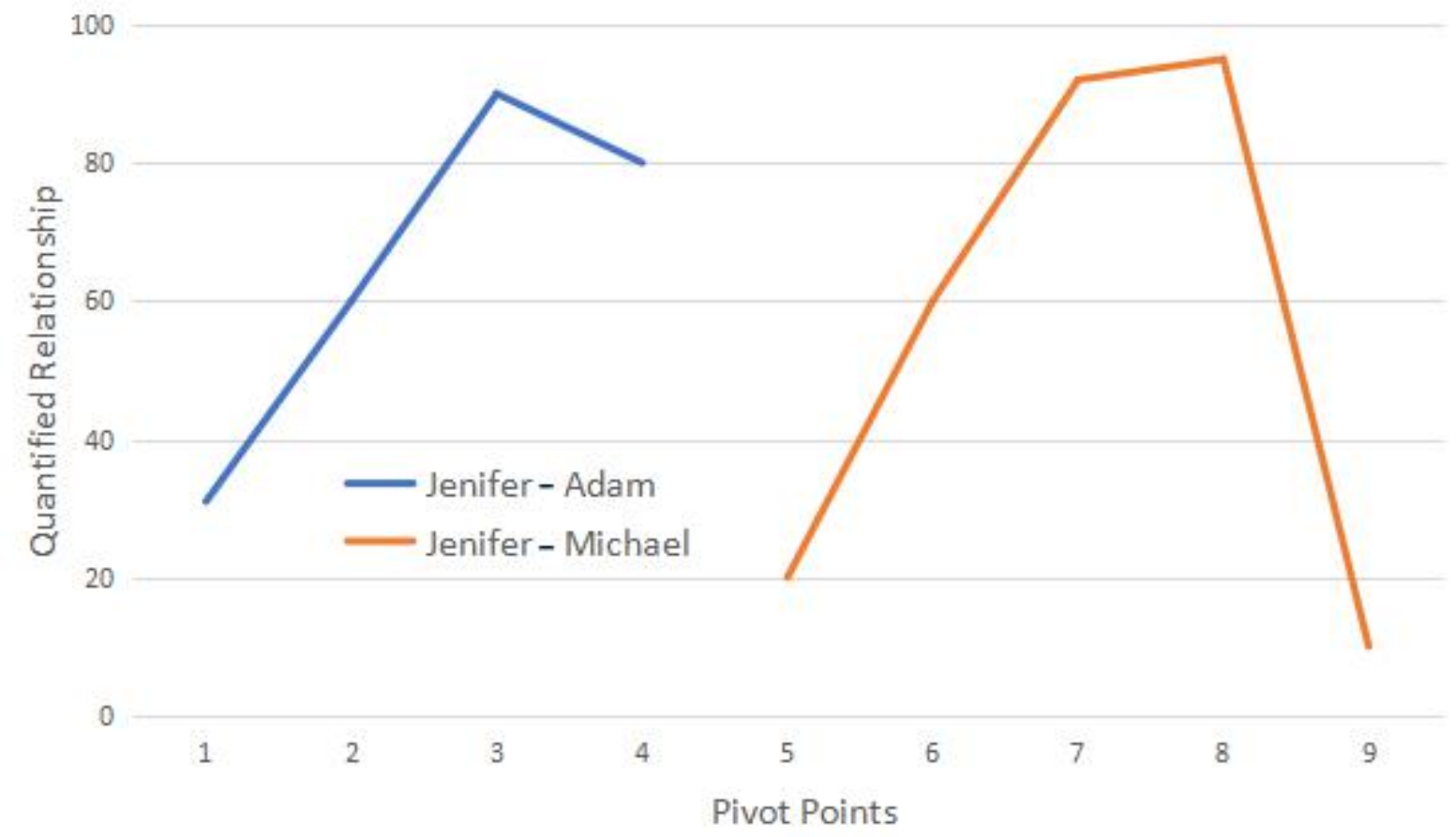

Figure 4: Character relationship progression in Rage of Angels for Jenifer and Adam (blue) and Jenifer and Michael (orange).

\section{Conclusion}

The cultural associations among candidates along with their personality and behavior impact on their literary inclination. Readers love narratives not just because of the language and story but also because of progression of relationships among characters in the narrative. This paper proposed an approach of mapping this progression to readers using graphs (Relationship Progression Analysis based on graph - RPAG). The results are analyzed with reference to data collected 150 book lovers, but the method may be scale to the analysis of thousands of candidates. We considered 50 of them for two classic fiction books. The accuracy observed is around 87 percent, which is consistent with verification by expert readers. Multiple characters are also considered in calculating this progression.

The algorithm can further be improved with multi-level graph clustering and filtering to handle relationships among different characters and their progression. The promising result and myriad uses of cultural association with literary inclination definitely help in taking new pathways for deliberation on further application specific methods. This method can find its applications in to identifying the best narrative for the reader, calculating possible prospects of a particular movie or a book. It can even evolve to application that can help publishing houses to classify and rank submitted manuscript before those are send to evaluators. Further research on single core character novels like "Fly-away" can prove very challenging. Also extending this idea for non-fictions and news can also be interesting extension to research.

\section{Acknowledgments}

We thank the BDB book club and all book lovers who provided information and inputs. 


\section{References}

Aisopos, F., Papadakis, G., Tserpes, K., \& Varvarigou, T. (2012). Textual and contextual patterns for sentiment analysis over microblogs. 21st International Conference on World Wide Web (WWW '12 Companion). ACM, New York, NY, USA.

Althoff, T., Clark, K., \& Leskovec, J. (2016). Large-scale Analysis of Counselling Conversations: an application of natural language processing to mental health. TACL, 4, 463476.

Berendt, B. (2017). Text mining for news and blogs analysis. Encyclopedia of Machine Learning and Data Mining. Springer, Berlin. doi:10.1007/978-1-4899-7687-1_833.

Boyd-Graber, J. \& Seppi, K. (2019). Automatic Evaluation of Local Topic Quality. arXiv, 1905.13126.

Chaturvedi, S., Srivastava, S., Daume, H., \& Dyer, C. (2016). Modeling evolving relationships between characters in literary novels. AAAI, Phoenix, Arizona.

Chaturvedi, S., Goldwasser, D., Daume, H. (2016). Ask, and shall you receive? understanding desire fulfilment in natural language text. AAAI, Phoenix, Arizona

Chen, Y-C. \& Bansal, M. (2018). Fast Abstractive Summarization with Reinforce-Selected Sentence Rewriting. ACL, Melbourne, Australia.

Fotis, J., Buhalis, D. and Rossides, N. (2012). Social media use and impact during the holiday travel planning process. In "Information and Communication Technologies in Tourism", M. Fuchs, F. Ricci, and L. Cantoni (eds.), pgs 13-24. Springer, Berlin.

Henrich, J. (2015). Culture and Social Behavior. Current Opinion in Behavioral Sciences, 3, 84-89.

Iyyer, M., Guha, A., Chaturvedi, S., Boyd-Graber, J., \& Daume, H. (2016). Feuding families and former friends: unsupervised learning for dynamic fictional relationships. ACL, San Diego, California

Jiang, D., Luo, X., Xuan, J., Xu, Z.. (2017). Sentiment Computing for the News Event Based on the Social Media Big Data. IEEE Access, 5.

Kulkarni, H. \& Alicea, B. (2019a). Cultural association based on machine learning for team formation. arXiv, 1908.00234

Kulkarni, H. \& Marathe, M. (2019b). Machine Learning Based Cultural Suitability Index (CSI) for Right Task Allocation. IEEE International Conference on Electrical, Computer and Communication Technologies (IEEE ICECCT), Coimbatore, India.

Li, J., Jia, R., He, H., \& Liang, P. (2018). Delete, retrieve, generate: a simple approach to sentiment and style transfer. ACL, New Orleans, Louisiana.

Liu, B. (2010). Sentiment Analysis and Subjectivity. Handbook of Natural Language Processing. Taylor and Francis Group, Boca Raton, FL. 
Lund, J. (2018). Fine-grained Topic Models Using Anchor Words. Dissertation. Brigham Young University, Provo, Utah.

Mitchell, J., \& Lapata, M. (2008). Vector-based Models of Semantic Composition. Proceedings of the ACL Conference, Columbus, Ohio.

Mooij, M. \& Hofstede, G. (2011). Cross-Cultural Consumer Behavior: a review of research findings. Journal of International Consumer Marketing, 23, 181-192.

Narsimhan, K. \& Brazilay, R. (2015). Machine Comprehension with Discourse Relations. ACL Conference on Non-Linear Programming, 1253-1262.

Oard, D.W. \& Carpuat, M. et.al (2019) Surprise Languages: Rapid-Response Cross-Language IR. ACM NTCIR-14 Conference, June 10, 2019 Tokyo Japan.

Quan, C. and Ren, F. (2011). Selecting clause emotion for sentence emotion recognition. International Conference on Natural Language Processing and Knowledge Engineering, Tokushima, Japan.

Rosenthal, S., Andreas, J., McKeown, K., \& Rambow, O. (2012). Detecting Influencers in Written Online Conversations. Proceedings of the Second Workshop on Language in Social Media, 37-45.

Saad, S.M. \& Kamarudin S.S. (2013). Comparative analysis of similarity measures for sentence level semantic measurement of text. IEEE International Conference on Control System, Computing and Engineering, 90-94.

Sood, S.O., Churchill, E.F., \& Antin, J. (2012). Automatic identification of personal insults on social news sites. Journal of the American Society for Information Science and Technology, 63(2), 270-285.

Wang, L. \& Cardie, C. (2014). A Piece of My Mind: A Sentiment Analysis Approach for Online Dispute Detection. ACL, Baltimore, Maryland. 